\title{
Linking theory and practice in teacher training: pandemic lessons from three countries (Russia, Kazakhstan and Slovakia)
}

\author{
[Reliant la théorie à la pratique dans la Formation des enseignants : les \\ leçons de la pandémie des trois pays (la Russie, le Kazakhstan et la \\ Slovaquie)]
}

\author{
Tatiana A. Baklashova - Kulzhanat N. Bulatbayeva - Juan Jose Mena Marcos - Peter \\ Plavcan
}

DOI: $10.18355 / X L .2021 .14 .04 .13$

\begin{abstract}
The objective of this comparative study devoted to the theoretical and practical training of future teachers (as e.g. French as a foreign language) in the context of Covid-19 is conditioned by the challenge that the pandemic represents for social institutions, by the global trends of the evolution of teacher training, by national priorities in the implementation of professional training in Russia, Kazakhstan, Slovakia as well as by the institutional educational strategies of the universities of the above-mentioned countries (Federal University of Kazan, National University Eurasian named after LN Goumilev and Comenius University of Bratislava). In this article, we analyze the institutional contexts of the training of future French teachers under the conditions of Covid-19 and from the point of view of changes in content and procedural aspects as well as the evaluative component of their professional training. The research priority was to assess the influence of pandemic conditions on the quality of the training of future French teachers and on the degree of training of their teaching skills, as well as to identify and justify a certain number of principles of the future training of French teachers. The study is based on systemic and task-based approaches. Research data was provided by 20 teachers and 44 university students from Russia and Kazakhstan; and by 11 students from Comenius University in Bratislava. The authors performed a reflexive-systematic analysis of educational activity and a qualitative factor analysis of unstructured interviews. The research results indicate the potential of integrating the theoretical and practical training of a future teacher of French in a pandemic environment, respecting a number of principles: 1) modularity; 2) polymodality (multimedia); 3) continuity of the contents of disciplines and practices; 4) dialogue between educational actors; 5) combination of assessment tools from theoretical and practical disciplines. The transition from face-to-face to distance training as well as the presence of gaps in digital teaching constitute a particular challenge in the training of future French teachers. The results of the study can be used for the development of educational programs for the training of French teachers and the implementation of joint research in the field of comparative pedagogy.
\end{abstract}

Key words: french, pandemic, teaching, technology, evaluation, continuity, modularity, polymodality, interaction

\begin{abstract}
Résumé
L'objectif de cette étude comparative consacrée à la formation théorique et pratique du futur professeur de FLE (français langue étrangère) dans le contexte du Covid-19 est conditionné par le défi que représente la pandémie pour les institutions sociales, par les tendances mondiales de l'évolution de la formation des enseignants, par les priorités nationales dans la mise en œuvre de la formation professionnelle en Russie, au Kazakhstan, en Slovaquie ainsi que par les stratégies éducatives institutionnelles des universités des pays susmentionnés (Université fédérale de Kazan, Université
\end{abstract}


nationale eurasienne nommée d'après L.N. Goumilev et Université Comenius de Bratislava). Dans cet article, nous analysons les contextes institutionnels de la formation des futurs enseignants de français dans les conditions du Covid-19 et du point de vue des changements du contenu et des aspects procéduraux ainsi que de la composante évaluative de leur formation professionnelle. La priorité de la recherche a été d'évaluer l'influence des conditions pandémiques sur la qualité de la formation du futur enseignant de français et sur le degré de formation de ses compétences pédagogiques, ainsi que d'identifier et de justifier un certain nombre de principes de la future formation des enseignants de français. L'étude est basée sur des approches systémique et actionnelle. Les données de la recherche ont été fournies par 20 enseignants et 44 étudiants universitaires de Russie et du Kazakhstan ; et par 11 étudiants de l'Université Comenius de Bratislava. Les auteurs ont effectué une analyse réflexive-systématique de l'activité pédagogique et une analyse factorielle qualitative d'entretiens non structurés. Les résultats de la recherche indiquent le potentiel de l'intégration de la formation théorique et pratique d'un futur enseignant de FLE dans un environnement pandémique, en respectant un certain nombre de principes : 1) modularité ; 2) polymodalité (multimédia) ; 3) continuité des contenus des disciplines et des pratiques ; 4) dialogue entre les acteurs éducatifs ; 5) combinaison des outils d'évaluation des disciplines théoriques et pratiques. Le passage du mode de formation en présentiel à celui à distance ainsi que la présence de lacunes en matière de didactique numérique constituent un défi particulier de la formation des futurs enseignants de français. Les résultats de l'étude peuvent être utilisés pour le développement de programmes éducatifs pour la formation des enseignants de français et la mise en œuvre de recherches conjointes dans le domaine de la pédagogie comparative.

Mots clé : français, pandémie, enseignement, technologie, évaluation, continuité, modularité, polymodalité, interaction

\section{Introduction}

La pandémie du Covid-19 a considérablement transformé le système de formation initiale des futurs enseignants (dont ceux de FLE) dans différents pays du monde, conférant une complexité particulière à la question de l'intégration de la théorie et de la pratique dans la mise en œuvre de la formation des enseignants (Assunção Flores , Gago, 2020; Ersin , Atay, 2021; La Velle et al., 2020). Par conséquent, la recherche comparative dans le domaine de la formation pédagogique, qui permet d'identifier les algorithmes (méthodologie) d'organisation du processus éducatif pour cette catégorie de spécialistes, revêt une grande importance. La méthodologie de l'organisation de la formation pédagogique nous permet de : 1. répondre à la question de l'évaluation de l'impact des conditions pandémiques sur la qualité de la formation des enseignants de français et sur le degré de formation de leurs compétences pédagogiques ; 2. identifier et justifier un certain nombre de principes de leur formation professionnelle. Cet article présente les résultats intermédiaires d'une étude comparative des chercheurs de trois universités de Russie, du Kazakhstan et de Slovaquie (l'Université fédérale de Kazan, l'Université nationale eurasienne nommée d'après L.N. Goumilev et l'Université Comenius de Bratislava), qui a débuté en janvier 2021.

Les mesures d'impact de la pandémie sur divers domaines sociaux et économiques de la société mondiale ont commencé en 2020 à être traitées non seulement par les Étatsnations, mais également par les organisations internationales mondiales.

L'Organisation de coopération et de développement économiques, basée à Paris, a publié le document «L'économie sociale et la crise du COVID-19 : défis actuels et futurs» en 2020, qui définit la région préopératoire recommandée. Ces mesures recommandées incluent la recommandation de définir une vision commune pour 
l'avenir, d'élaborer un plan d'action pour réaliser la transition vers un modèle de développement durable et plus inclusif, et de soutenir après 2020.

L'Association internationale des universités (AIU) a également mené une enquête mondiale en 2020 sur l'impact des mesures en cas de pandémie dans les États-nations sur l'offre d'enseignement supérieur. Plus de quatre cents universités de 109 pays sur tous les continents participent à l'enquête. Dans l'étude publiée «Impacts du COVID19 sur les universités dans le monde», plusieurs autres résultats ont été présentés (Marinoni, Land, Jensen, 2020) :

- La COVID-19 a touché presque toutes les institutions qui ont répondu au sondage. $59 \%$ d'entre eux ont déclaré que toutes les activités du campus avaient été arrêtées et que l'établissement était complètement fermé.

- Presque toutes les universités $(91 \%)$ ont une infrastructure en place pour communiquer avec leurs étudiants et leur personnel au sujet de la COVID-19. Néanmoins, les répondants ont immédiatement appelé pour assurer des flux de communication clairs et efficaces avec le personnel et les étudiants.

- Dans presque toutes les universités, l'évolution de la COVID-19 a influencé l'enseignement et l'apprentissage, les deux tiers signalant que l'enseignement en classe a été remplacé par l'apprentissage à distance. La transition de l'enseignement à temps plein à l'enseignement à distance ne s'est pas faite sans heurts. Les principales lacunes comprenaient l'accès à l'infrastructure technique, aux compétences et à la pédagogie pour l'apprentissage à distance et les exigences de domaines d'études spécifiques.

- La transition forcée vers l'apprentissage et l'apprentissage à distance offre des opportunités importantes pour concevoir des options d'enseignement plus flexibles, explorer l'apprentissage mixte ou hybride et combiner l'apprentissage synchrone avec l'apprentissage asynchrone.

- Dans le même temps, 60 \% des universités ont indiqué que le programme COVID19 a augmenté la mobilité virtuelle et/ou l'apprentissage en ligne conjoint comme alternative à la mobilité physique des étudiants. Cela peut assurer l'internationalisation dans une certaine mesure, mais ce changement devra être analysé plus en détail, selon les auteurs de l'enquête.

Ayant pour but d'identifier des mécanismes permettant d'assurer l'intégration de la théorie et de la pratique dans le contexte d'un enseignement à distance pendant la pandémie, l'étude vise à déterminer les changements en termes de contenu et de technologie, ainsi qu'à identifier l'évaluation des résultats de la formation professionnelle des futurs enseignants de français. La priorité de l'étude est d'évaluer l'impact des conditions pandémiques sur la qualité de la formation du futur professeur de FLE (Auger, Louis , Dervin, 2009), sur le niveau de formation des compétences didactiques : maîtrise de la langue en tant que système (variétés structurelles et fonctionnelles), bases didactiques de sélection du matériel pédagogique (du point de vue de la langue et de la parole), technologies numériques d'enseignement efficaces, compétences de gestion de l'organisation du travail, etc. Il était important d'identifier et de justifier un certain nombre de principes de la formation professionnelle des futurs enseignants de français, à savoir : le principe de modularité, permettant la flexibilité et la variabilité des programmes ; le principe de multimodalité (multimédia) de la formation, permettant l'utilisation dans le processus d'apprentissage des modes de perception visuelle, auditive et motrice ; le principe de cohérence pour la continuité du contenu des disciplines théoriques et des pratiques pédagogiques; le principe de dialogue, permettant une interaction efficace et la communication de tous les acteurs du processus éducatif ; enfin, le principe de combinaison des outils d'évaluation des disciplines théoriques et pratiques. Les questions de recherche étaient les suivantes: 1. Quels sont les effets de l'influence des conditions pandémiques sur la sphère de la formation professionnelle-pédagogique et théorique-pratique du futur professeur de FLE en Russie et au Kazakhstan ? 2. Quels sont les avantages de la formation pratique des futurs enseignants réalisée à distance (en comparaison des contextes de 
préparation de l'enseignant de français dans les universités des trois pays) ; les effets positifs de la réalisation des disciplines théoriques et de la préparation pratique de l'enseignant selon les principes révélés et fondés de modularité des contenus de l'enseignement pédagogique, de polymodalité (multimédia) de formation, de continuité des contenus de la préparation pratique du futur enseignant, de dialogue entre les acteurs du processus éducatif et de combinaison des outils d'évaluation des disciplines théoriques et pratiques?

Cet article est composé des sections suivantes: introduction, étude bibliographique, méthodologie, résultats et discussion, conclusion, remerciements et références.

\section{Etude bibliographique}

L'étude bibliographique montre que l'intégration de la théorie et de la pratique dans le processus éducatif est aujourd'hui un défi particulier, qui était déjà assez prononcé dans le monde pré-Covid-19 (Gao , Zhang, 2020). Les systèmes de formation des enseignants du monde entier ont également été confrontés au défi de perturber les liens intégratifs habituels entre la théorie et la pratique dans le cadre de l'enseignement à distance en raison de la pandémie (Mishra, Gupta , Shree, 2020), à celui du changement de mode de communication, de dialogue intégral avec les acteurs de la formation pratique et théorique du futur enseignant (passage à la communication en ligne) (Erlam , Ellis, 2018), et enfin au défi de l'émergence de la nécessité d'une mise en œuvre de qualité de pratiques diverses dans des conditions d'accès hors ligne limité aux laboratoires pratiques, aux bases de pratique, etc. Il est urgent de développer et de tester différentes methodologies de didactiques numériques (Trubavina et al., 2021) pour former de manière compétente les élèves-enseignants dans un environnement d'apprentissage à distance. Parallèlement aux difficultés d'organisation de la formation théorique et pratique des enseignants dans la mise en œuvre du format d'apprentissage à distance, nous pouvons mettre en avant les avantages de la transition globale vers le numérique. Ainsi, un certain nombre d'études et de documents analytiques indiquent que l'enseignement à distance n'a pas affecté le niveau du rendement des étudiants ni leur maîtrise des compétences de l'activité professionnelle et pédagogique (Kosyakova , Popova, 2020; Aslanyan, 2021). La modalité à distance a donné lieu à une plus grande mobilité des étudiants, les a libérés de leurs attaches à leur lieu d'étude et leur a offert un mode d'apprentissage individuel avec la possibilité d'écouter en différé des cours magistraux enregistrées (Shahrill et al., 2021). Cette modalité leur a également permis de développer des compétences numériques et d'acquérir des connaissances en informatique ; elle leur a aussi permis d'amorcer leurs processus d'auto-apprentissage et d'auto-développement (Murphy, Eduljee , Croteau, 2020).

Dans ce contexte d'éducation en ligne de masse en situation de pandémie, la question de la formation professionnelle théorique et pratique d'un futur professeur de FLE, conçue pour répondre aux besoins de l'État et de la société, revêt une importance particulière dans les travaux des chercheurs (Benito Pérez, 2020; Kaspary , De sousa, 2020; Mikhailova et al., 2021). L'enseignant se voit aujourd'hui chargé de tâches socialement significatives (Babenko et al., 2017) : il agit comme intermédiaire actif entre les acteurs du processus éducatif, notamment la famille, l'établissement scolaire et les autorités éducatives. Dans le domaine de l'activité des pédagogues, des problèmes d'organisation se posent lorsqu'il s'agit de fournir aux étudiants des moyens d'apprentissage en ligne, d'offrir un soutien aux parents d'élèves ainsi que des conseils en ce qui concerne l'aide aux enfants dans le cadre de l'apprentissage à distance, de réaliser les activités de mentorat (Usak, Masalimova, 2019), ou d'assurer le confort psychologique des étudiants en cas de pandémie (Morvan , Frajerman, 2021). En plus des tâches socialement significatives, le professeur de FLE doit couvrir des objectifs méthodologiques professionnellement significatifs. Ceux-ci sont associés à la maîtrise de compétences linguistiques, culturelles et méthodologiques

XLinguae, Volume 14 Issue 4, October 2021, ISSN 1337-8384, eISSN 2453-711X 
(Solovyeva, Sabirova , Morozova, 2015; Saadatnejad , Farsian, 2020), y compris la didactique numérique, constitutives de sa capacité professionnelle fonctionnelle (Serostanova , Choporova, 2020). Aujourd'hui, la priorité est de maitriser: - les compétences d'utilisation productive des technologies numériques (Gartsov, 2009); la capacité de prendre en compte les besoins individuels des élèves de français, compte tenu de la présence ou de l'absence d'un environnement francophone (Bulatbaeva, Sivakova , Galiakhmetova, 2017); - la capacité de sélectionner le matériel lexical et grammatical de formation, d'appliquer différentes techniques d'enseignement basées sur des textes dialogiques et monologiques, et d'utiliser différentes méthodologies d'enseignement communicatif (Fahrutdinova et al., 2018). L'ensemble des tâches socialement et professionnellement significatives d'un enseignant contemporain de FLE exerçant son activité pédagogique dans des conditions de pandémie, met en évidence les éléments suivants: 1. l'application de cours de formation sur la pratique de l'enseignement du français dans le format de l'enseignement en ligne, suivie d'une réflexion collective sur les leçons de l'auteur menée par les étudiants; 2. l'accent mis sur le développement de la personnalité créative d'un futur enseignant (Bovtenko, 2005) ; 3. la mise en œuvre de la formation pédagogique d'un enseignant de langue étrangère, impliquant la maîtrise de cette dernière dans la triptyque: moyen de communication (Kavilova, 2020), objet d'apprentissage, et instrument d'apprentissage et de communication pédagogique (Efimkina, 2010).

Les circonstances susmentionnées mettent au jour le problème de la recherche d'une nouvelle méthodologie d'organisation de la formation professionnelle d'un futur professeur de FLE dans un établissement d'enseignement supérieur. Cette formation implique une intégration obligatoire de la théorie et de la pratique dans le cadre du passage à l'enseignement à distance. Il est important d'identifier les priorités dans le développement des contextes institutionnels de l'enseignement des langues dans différents pays dans les conditions de propagation du Covid-19 (Cutri, Mena , Whiting, 2020), de déterminer les principes de la formation professionnelle théorique et pratique des enseignants de langues étrangères, d'identifier les changements dans les aspects de contenu et de procédure, ainsi que l'évaluation des résultats de l'apprentissage. Ainsi, la création de programmes d'études plus flexibles (principe de modularité) (Nozdrevatykh , Nozdrevatykh, 2020), la garantie de la continuité du contenu des disciplines et des pratiques (principe du caractère systématique) (Somkin, 2008), le dialogue des acteurs du processus éducatif (principe du dialogue) (Kostikova, 2008), ainsi que la combinaison des outils d'évaluation des disciplines et des pratiques (principe d'interrelation) (Griftsova , Linkov, 2018) sont considérés comme prioritaires dans le contexte russe. La priorité pour le contexte éducatif du Kazakhstan consiste en une large application des supports multimédia à la formation des enseignants de français, ce qui implique la présentation d'informations par le biais de diverses plateformes médiatiques, de moyens (textes verbaux, matériel photo et vidéo, graphiques, animation, etc.) (principe de polymodalité ou multimédia (Podymova, Golovyatenko, Khmelkova, 2021). La construction du processus éducatif sur la base du principe de polymodalité permet de fournir une perception polymodale du matériel éducatif (modes de perception visuels, auditifs, moteurs) (Uribe Guinard, 2018). La perception polymodale permet de connaître les objets et les phénomènes de manière complexe et de former des images holistiques (Kapitsa, 2013).

Ces priorités définissent le vecteur de transformation de l'organisation, du contenu, des technologies et de l'évaluation des résultats de la future formation des enseignants et précisent les changements (Sánchez-García, GuanLin , Escribano, 2013) comme suit :

I. Sur le plan organisationnel : - la mise à jour du contenu de la formation en tenant compte des nouvelles conditions (formation en ligne en raison de la pandémie, numérisation globale, enseignement centré sur la science) ; - l'amélioration des 
modèles de partenariat école-université (Menter, Valeeva , Kalimullin, 2017), - le renforcement systémique de l'institut de formation initiale des enseignants.

II. Sur le plan du contenu et du processus : - l'architecture modulaire des programmes d'études ; - la logique d'organisation des stages à temps plein et à temps partiel mis en œuvre dans le cadre du partenariat école-université (lycées de la KFU) (Baklashova, Skobeltsyna , Galishnikova, 2020); - l'ensemble des formats d'apprentissage interactifs (formations, master classes, sessions de conception, réunions de discussion sur la recherche, travaux pratiques en classe de simulation, apprentissage en ligne), ce qui permet de faire correspondre avec succès la théorie et la pratique dans la formation de l'enseignant du $21^{\text {ème }}$ siècle dans les conditions d'une université classique (Valeeva, Gafurov, 2017; Gafurov et al., 2018; Valeeva, Kalimullin, 2019; Baklashova et al., 2020).

III. Sur le plan de l'évaluation complexe du niveau de formation des compétences : - la combinaison du fonds d'outils d'évaluation (FOE) des disciplines théoriques et des pratiques ; - l'utilisation de tâches professionnelles (cas, développés en vue des exigences scolaires contemporaines) pendant l'attestation d'Etat de fin d'études dans l'établissement d'enseignement supérieur; - la participation à la conception du FOE, des procédures d'évaluation des résultats de l'apprentissage par les employeurs, les mentors des écoles ou les tuteurs de l'Université.

Les priorités susmentionnées du développement des systèmes russe et kazakh de formation des enseignants dans le contexte de la pandémie, ainsi que les orientations vectorielles de la transformation de l'organisation, du contenu, des technologies et de l'évaluation des résultats de la formation professionnelle des futurs enseignants de FLE en mettant l'accent sur l'intégration de la théorie et de la pratique dans l'enseignement, exigent qu'une recherche scientifique soit menée afin d'évaluer la signification des stratégies et des modèles éducatifs élaborés et mis en œuvre dans la pratique. La présente étude est un exemple de l'une de ces études.

\section{Méthodologie}

Les auteurs ont utilisé des approches systémiques et basées sur les activités (Nomokonova , Tsvetkov, 2015; Sheptukhovsky, 2012). La première d'entre elles, qui est une méthode de recherche universelle, est fondée sur la perception de l'objet étudié comme un tout unique, composé de parties interdépendantes et faisant simultanément partie d'un système d'ordre supérieur (Khasanova, 2021). L'approche systémique permet d'identifier les propriétés intégratives du système et les caractéristiques qualitatives qui sont absentes des éléments qui le composent. Appliquée à la recherche dans le domaine de l'éducation, l'approche systémique offre la possibilité de pénétrer plus profondément dans l'essence du processus éducatif, d'établir des relations entre les problèmes pédagogiques, d'obtenir des informations plus objectives sur le mécanisme du processus d'éducation de la personnalité de l'enseignant, de différencier les relations dans le système éducatif et, par ce biais, de créer une vision holistique de celui-ci. La deuxième approche, basée sur l'activité, implique une recherche dans le processus réel d'interaction des acteurs du processus éducatif (dans notre cas, la formation des enseignants de français), ce qui nous permet de garantir la solution de certaines tâches professionnellement significatives.

Les données de cette recherche ont été fournies par 20 enseignants de l'enseignement supérieur impliqués dans le processus de formation des futurs enseignants de français : 10 interrogés appartenant au corps enseignant de l'université fédérale de Kazan (Kazan, Russie) et 10 interrogés du corps enseignant de l'université nationale eurasienne nommée d'après L.N. Goumilev (Nour-Soultan, Kazakhstan) ; 44 étudiants (22 interrogés appartenant au corps étudiant (niveau master) de l'Université fédérale de Kazan (Kazan, Russie) et 22 étudiants du corps étudiant de l'université nationale eurasienne nommée d'après L.N. Goumilev (Nour-Soultan, Kazakhstan).

XLinguae, Volume 14 Issue 4, October 2021, ISSN 1337-8384, eISSN 2453-711X 
L'analyse des matériaux factuels obtenus (étude de cas du Kazakhstan) a été effectué dans le laboratoire de recherche de l'Académie nationale de l'éducation nommée d'après Y. Altynsarin (Nour-Soultan, Kazakhstan).

Les auteurs ont effectué une analyse du sujet de recherche basée sur la littérature scientifique, l'analyse réflexive-systématique de l'activité professionnelle et pédagogique, et l'analyse qualitative factorielle des entretiens non structurés avec les participants au processus éducatif. La méthode des entretiens en profondeur a été choisie comme principale méthode. Cette méthode renvoie aux méthodes d'enquête de la recherche pédagogique et est utilisée dans une plus large mesure pour interroger des spécialistes travaillant dans un domaine professionnel particulier. Les entretiens en profondeur (non formalisés) de cette étude ont été réalisés au début du deuxième semestre de l'année universitaire 2020/2021. Les réponses des participants ont été enregistrées par l'enquêteur. La tâche principale lors de la conduite d'entretiens en profondeur était de gérer d'organiser une communication professionnelle-pédagogique intentionnelle et prédéterminée entre l'enquêteur et le répondant. Il convient de souligner que le choix de cette méthode n'est pas accidentel et se justifie par sa flexibilité et sa capacité à obtenir des informations subjectives et comportementales. La méthode des entretiens en profondeur a permis d'organiser des entretiens individuels, libres et directs avec les interrogés. Au cours de conversations individuelles et non structurées d'une heure, l'enquêteur a pu connaître l'opinion personnelle de chaque répondant sur les questions faisant l'objet de la recherche. Il est important de souligner que les interrogés ont exprimé leurs points de vue de manière assez libre et complète, en répondant aux questions des enquêteurs, ce qui n'est pas toujours le cas dans les discussions tenues en public. Nous avons observé une attitude plutôt sérieuse des répondants à l'enquête favorisée par la nature individuelle des entretiens. Les enquêteurs ont pu adapter les techniques d'entretien en profondeur aux conditions de la situation spécifique, pour contrôler les informations détaillées reçues sur les opinions, les perceptions du répondant, puisque les chercheurs se concentraient sur une seule personne à chaque fois. Ils ont pu observer et prendre en compte les réactions non-verbales du répondant lors de l'évaluation, et adapter le déroulement de l'entretien si nécessaire.

Dans le cadre des entretiens en profondeur, l'enquêteur a d'abord présenté à la personne interrogée le contexte du problème, en soulignant l'impact de la pandémie de la Covid-19 sur les institutions sociales, y compris les établissements d'enseignement supérieur. Ensuite, au cours d'une conversation informelle, le répondant a été invité à répondre à plusieurs questions clarifiant sa position, son évaluation personnelle des effets des conditions pandémiques sur la sphère de l'éducation, le système de formation pratique des enseignants dans les établissements d'enseignement supérieur, sur le niveau de maîtrise des compétences professionnelles et pédagogiques.

$\mathrm{Au}$ cours de l'entretien mené par l'enquêteur, le problème de la mise en œuvre de la formation théorique et pratique du futur professeur de FLE dans les conditions de la quarantaine universelle a été mis au jour, les caractéristiques, les difficultés de la formation pratique dans un format à distance, le potentiel des technologies numériques ont été soulignés, les défis de la formation pratique du futur professeur de français en lien avec la propagation de la Covid-19 ont fait l'objet d'une exposition de la part de l'enquêteur. Il a été demandé aux répondants d'évaluer le succès du processus de formation théorique et pratique à distance, la complicité, le soutien de tous les acteurs du processus de pratique pédagogique à l'université, et de citer les principales difficultés et les avantages de la mise en œuvre des pratiques dans le contexte de la pandémie.

En ce qui concerne le contenu de l'enseignement, l'entretien a porté sur la modularité des programmes d'études, qui permet de faire correspondre, d'intégrer la théorie et la pratique. L'enquêteur a souligné les difficultés liées à la sélection du contenu de la formation théorique et pratique des enseignants, s'est concentré sur la logique de la 
construction du processus éducatif, et a souligné l'importance de la compétence scientifique et méthodologique dans la sélection du contenu éducatif. Les interviewés ont répondu à des questions concernant l'évaluation des avantages des plans modulaires par rapport aux plans réguliers, ainsi que la combinaison de disciplines théoriques et de travaux pratiques.

Les entretiens ont également abordé le problème de la continuité du contenu des pratiques dans l'enseignement à distance en raison de la pandémie. Les participants aux entretiens se sont vus expliquer les particularités de l'architectonique des programmes institutionnels des programmes de magistrature pédagogique, notamment : l'absence de duplication des tâches pratiques, le lien avec les disciplines théoriques du module et la conformité du contenu pratique avec le contexte éducatif moderne. Les répondants ont été invités à évaluer le degré de continuité dans le contenu des pratiques, la pertinence des travaux pratiques ainsi que leur lien avec les réalités de l'école moderne. Il était également important d'évaluer l'attitude des participants à l'étude face à l'évaluation des résultats de la pratique et à la procédure de protection mise en œuvre dans un format virtuel.

Un accent particulier a été mis sur le problème de l'interaction entre les participants du processus de formation pratique. L'enquêteur a expliqué que le processus d'interaction et de communication est complexe et multiforme. Il a notamment souligné que l'interaction joue un rôle important dans le processus éducatif car, sans elle, la mise en œuvre de l'éducation est impossible. Les interrogés devaient répondre à des questions posées dans l'ordre suivant : Parvenez-vous à mener un dialogue productif avec tous les acteurs de la formation pratique ? Quelles sont les difficultés que vous rencontrez ? Comment la pandémie a-t-elle affecté le niveau de communication dans la pratique ? Compte tenu des caractéristiques positives de cette méthode, nous estimons qu'il convient de souligner aussi ses inconvénients rencontrés au cours de l'étude : 1) la nécessité obligatoire pour les chercheurs d'atteindre le niveau de compétence d'un enquêteur hautement qualifié, ce qui est un processus assez chronophage ; 2) la présence d'un certain risque de réduction de la qualité et de la profondeur des informations obtenues auprès du répondant sous l'influence de l'enquêteur ; 3) une consommation de temps élevée (75 heures au total) ; 4) des procédures chronophages de collecte et d'analyse des données et de l'information.

\section{Résultats et Discussion}

\section{Le cas de l'Université fédérale de Kazan (région de la Volga)}

Les résultats suivants ont été obtenus au cours de l'étude.

1. Selon le corps des enseignants de l'université :

$60 \%$ des répondants signalent un impact assez fort de la pandémie sur l'éducation, y compris sur le système de formation théorique et pratique du personnel enseignant. Le passage à un format d'apprentissage à distance pour de nombreux collègues (40\%) a été associé à l'émergence d'un besoin aigu d'améliorer leurs compétences en matière de TIC, ainsi qu'à la possibilité d'un développement actif dans le domaine de l'éducation numérique $(70 \%)$.

Répondant $\mathrm{n}^{\circ} 5$ :

"Notre progression dans la mise en cuvre de l'éducation numérique a été rapide... Il $y$ avait un certain nombre de difficultés, mais le fait que, dès le début de l'introduction de l'enseignement à distance dans un environnement pandémique, la direction de l'Université ait décidé d'utiliser une seule plateforme Microsoft Teams pour mettre en cuvre le cours des conférences, des séminaires, l'organisation de divers genres et types de pratique, a beaucoup aidé. En outre, il est devenu très simple de réunir le corps étudiant en équipes pour discuter des questions problématiques du processus éducatif. Nous avons gagné du temps et avons pu agir rapidement».

Répondant $\mathrm{n}^{\circ} 2$ :

XLinguae, Volume 14 Issue 4, October 2021, ISSN 1337-8384, eISSN 2453-711X 
"Pendant a période d'enseignement à distance en raison de la pandémie, nous avons progressé avec les étudiants. Souvent, ils étaient en avance sur leur temps et nous soutenaient pleinement dans la maîtrise des nouveaux outils numériques $\gg$.

Répondant $\mathrm{n}^{\circ} 8$ :

«La construction de cours par voie numérique a exigé une révision des approches méthodologiques classiques que j'ai mises en ouvre pendant des décennies dans l'enseignement supérieur. Il est devenu beaucoup plus difficile de maintenir l'attention des élèves. Dans un souci d'efficacité des cours pratiques à distance, nous devons constamment nous préoccuper du retour d'information que nous recevons des étudiants. Trouver et tester de nouvelles méthodes de travail dans des formats numériques est l'une des tâches du développement de la didactique numérique ».

Ainsi, il est raisonnable de faire état de l'impact significatif de la pandémie sur le niveau de compétence en TIC des participants au processus éducatif ; de la qualité de l'organisation de l'enseignement à distance pour les enseignants sur une plateforme numérique professionnelle adaptée et orientée vers l'apprentissage ainsi que de la méthodologie de l'organisation de la formation professionnelle et pédagogique des futurs enseignants à l'université.

$50 \%$ des répondants ont mentionné l'importance des ressources pédagogiques numériques (RPD) dans l'enseignement théorique et pratique des futurs professeurs de FLE. Ces ressources (RPD) ont été développées bien avant l'apprentissage à distance en raison de la pandémie et couvrent la plupart des disciplines théoriques et des pratiques d'orientation différentes. $20 \%$ des répondants ont souligné les difficultés d'interaction avec le corps étudiant lors de l'organisation et de la mise en œuvre du processus de formation pratique dans un environnement d'enseignement à distance, tandis que $50 \%$ a évalué positivement les possibilités de communication vidéo opérationnelle avec le groupe lors de l'utilisation des applications Web de réunion et de conférence. $80 \%$ des enseignants ont souligné l'importance du format « live » de la formation.

Les programmes d'études modulaires ont fait l'objet d'une évaluation très positive. Les répondants ont particulièrement noté : la logique des programmes d'études $(70 \%)$, la continuité des disciplines théoriques ainsi que les stages au sein du programme de maîtrise $(60 \%)$. L'approche méthodologiquement correcte consistant à associer les disciplines théoriques et les tâches de stage est jugée positive par $70 \%$ des enseignants interrogés et des superviseurs de stage des universités et des écoles.

Répondant $\mathrm{n}^{\mathrm{O}} 3$ :

"L'intégration du contenu des disciplines théoriques et des pratiques présente de nombreux avantages: les étudiants se rendent compte de l'importance et de la nécessité des connaissances théoriques par rapport à la pratique de l'activité pédagogique; les responsables de la pratique (mentors, tuteurs) et les enseignants qui donnent les cours ont la possibilité de prendre des décisions conjointes sur le contenu, sur les technologies des activités éducatives, ainsi que sur le système d'évaluation des compétences professionnelles et pédagogiques formées ; l'évaluation des résultats de l'apprentissage, la période de pratique à l'école est systémique et permet d'évaluer le niveau de compétence ».

Il faut souligner que les conversations menées avec les enseignants-animateurs des pratiques, ont permis d'identifier les problèmes de leur interaction personnelle et professionnelle avec le corps étudiant pendant la mise en œuvre de la formation pratique, ainsi que la nécessité de la mise en œuvre de la formation dans un format «live» comme une condition nécessaire au dialogue des acteurs du processus éducatif. La modularité des programmes d'études a facilité l'intégration efficace de la théorie et de la pratique dans le processus éducatif. Cette intégration a permis de surmonter le passage de la théorie à la pratique, d'optimiser les processus de communication et de surmonter la contradiction entre les connaissances théoriques et la mise en œuvre des actions dans la pratique. 
2. Selon le corps d'étudiants de l'université (étudiants en master) :

La majorité des étudiants interrogés $(90,8 \%)$ a noté que la pandémie a eu un impact significatif sur le domaine de l'éducation en général et sur celui de la formation des enseignants en particulier. Une proportion bien moindre des étudiants interrogés $(9,08 \%)$ a indiqué que la pandémie n'avait pas eu d'impact sur le secteur de l'éducation. Les répondants ont noté les effets positifs et négatifs du passage à la modalité à distance de la quarantaine en 2020. Parmi les effets positifs : une augmentation de la culture numérique $(54,48 \%)$, un renforcement des capacités en termes de compétences TIC $(40,86 \%)$ et une meilleure connaissance des applications liées à l'éducation $(49,94 \%)$. Les étudiants ont également souligné des avantages tels que le gain de temps $(63,56 \%)$, la possibilité de résoudre de nombreuses questions de procédure $(49,94 \%)$, d'étudier à leur domicile plus confortablement $(45,4 \%)$, de s'engager dans l'auto-éducation (31,78\%), et enfin de construire le mode d'étude de chacun de manière indépendante $(22,7 \%)$. Certains répondants $(13,62 \%)$ ont également noté le potentiel accumulé par l'université pour mettre en œuvre l'éducation numérique, ils ont souligné son importance dans la transition vers l'enseignement à distance en raison de la pandémie et ont identifié la priorité du développement de la didactique numérique dans l'université pour mettre en œuvre l'apprentissage axé sur les compétences.

Répondant $\mathrm{n}^{\circ} 9$ :

"Le passage à l'enseignement à distance était le seul moyen d'acquérir des connaissances. Grâce aux ressources numériques déjà disponibles, l'université a pu s'adapter rapidement aux nouvelles exigences et mettre en ouvre le programme éducatif».

"Les ressources numériques sont devenues une source majeure d'apprentissage pour nous. Pour maîtriser les compétences pédagogiques, l'utilisation des ressources numériques requiert non seulement la capacité d'utiliser les ressources numériques, mais aussi de les adapter au public, de les appliquer habilement conformément aux buts et objectifs de l'éducation ».

En plus des effets positifs, les répondants ont indiqué des effets négatifs de la pandémie sur le système éducatif : diminution de la motivation $(4,54 \%)$ et diminution de l'efficacité de l'enseignement $(13,62 \%)$; il a également été noté que la formation était freinée en raison du manque de compétence des enseignants en matière de TIC $(18,16 \%) .9,08 \%$ des répondants ont noté une détérioration de leur bien-être due à l'apprentissage à distance en cas de pandémie (baisse de la vision, maux de tête, effets négatifs sur le système musculosquelettique). $13,62 \%$ du nombre total de répondants a souligné que les étudiants étaient distraits par des soucis personnels pendant les séances en format virtuel, ce qui a interféré avec le processus d'apprentissage (travail en équipe, en binôme). $9,08 \%$ des répondants ont souligné que la formation des compétences professionnelles et pédagogiques en format virtuel est extrêmement difficile.

Répondant $\mathrm{n}^{\mathrm{o}} 14$ :

"L'enseignement à distance a été un défi que nous avons dû endurer. Nous n'avons pas eu le choix... Pour améliorer les compétences pédagogiques, le format virtuel, à mon avis, n'est pas adéquat ".

Ainsi, les évaluations du corps étudiant rejoignent celles des enseignants : on y retrouve un impact significatif de la pandémie sur le domaine de l'éducation, la présence d'effets tant positifs que négatifs de l'enseignement à distance, ainsi que les priorités pour le développement de la didactique numérique en tant que science et pratique. Une grande importance est accordée aux aspects d'autonomie dans l'apprentissage, d'auto-éducation, de plus grande mobilité, d'amélioration des compétences en matière de TIC.

XLinguae, Volume 14 Issue 4, October 2021, ISSN 1337-8384, eISSN 2453-711X 
Les effets négatifs de la distance pandémique ont également été clairement décrits par les personnes interrogées, lors des entretiens. Pour la plupart, les étudiants ont signalé des problèmes de santé ainsi que la perturbation du cours habituel des études en raison du niveau insuffisant de compétence numérique de l'enseignant ou du maître de stage. La difficulté de créer l'excellence pédagogique dans des conditions non présentielles a été particulièrement notée.

$68,1 \%$ des répondants ont souligné le succès du stage dans un format mixte, mais les mêmes répondants ont noté que le format d'apprentissage à distance ne remplacera jamais la communication « en direct », en face à face. $22,7 \%$ a déclaré qu'il n'y avait pas de difficultés dans la pratique. $63,56 \%$ des répondants ont relevé un niveau élevé d'interaction avec les responsables des pratiques de l'université et de l'établissement scolaire ainsi qu'un soutien compétent pendant le stage. 9,08\% a mentionné le problème bureaucratique pendant le stage et le changement des exigences concernant les documents de rapport de stage.

Répondant $\mathrm{n}^{\circ} 11$ :

"Les responsables de stage sont toujours prêts à répondre aux questions en temps voulu et de manière complète. Cependant, pendant le stage, j'aurais aimé avoir plus de réunions en ligne pour contrôler en continu le processus de l'établissement de documents ».

Répondant $\mathrm{n}^{\circ} 12$ :

"Les responsables de stage ont couvert les informations qui nous étaient nécessaires de manière exhaustive et nous ont informés en temps voulu. La tenue de conférences périodiques sur le stage à distance s'est avérée très appropriée ».

"En ce qui concerne le stage de recherche, nous avons eu beaucoup de chance. Le responsable était en contact avec nous tous les jours pour répondre à nos questions éventuelles".

Répondant $\mathrm{n}^{\circ} 20$ :

«Nous avons eu un cours avant le stage pendant lequel une analyse détaillée de notre travail à venir a été faite, grâce à quoi la réalisation du stage n'a posé aucune difficulté. L'avantage du format virtuel est l'économie de papier. La version électronique de la documentation du rapport de pratiques a été facilement corrigée sur Google drive ».

Répondant $\mathrm{n}^{\mathrm{o}} 16$ :

"Le dialogue avec le responsable est toujours parfait. Parfois, le travail en équipe pose des difficultés. Malgré les avantages du travail à distance, aucun service ne peut parfois remplacer la communication vivante ».

Lorsqu'on leur a demandé d'évaluer la modularité des programmes d'études permettant l'intégration de la théorie et de la pratique dans l'éducation, 59,02\% des interrogés ont exprimé une opinion positive à l'égard des programmes d'études modulaires, notant la logique de leur construction $(40,86 \%)$, ainsi que la coexistence de disciplines théoriques et de travaux pratiques $(31,78 \%)$. 45,4\% des répondants ont souligné la continuité des programmes de stage. $40,86 \%$ des répondants ont indiqué qu'ils étaient pleinement satisfaits de la procédure de soutenance des résultats du stage pratique, qui s'est déroulé à distance. $13,62 \%$ des étudiants participant à la recherche ont noté comme un avantage la possibilité de faire un stage dans l'établissement scolaire dans lequel ils travaillent habituellement.

Répondant $\mathrm{n}^{\circ} 9$ :

"J'aime beaucoup ce principe de conception du programme d'études. Les disciplines sont liées logiquement, ce qui facilite l'apprentissage de la matière ».

Répondant $\mathrm{n}^{\circ} 11$ :

"Les modules sont bons. Le niveau d'organisation du contenu de l'apprentissage est élevé. Le contenu est raisonnablement structuré. Une nette différence est ressentie par rapport aux programmes d'études conventionnels ».

Répondant $\mathrm{n}^{\circ} 13$ : 
"Il est très utile de relier les disciplines théoriques aux stages pratiques. Lorsque nous recevons une consigne d'un enseignant dans une discipline théorique, nous la mettons en pratique. Cette combinaison nous fait gagner du temps et nous permet de mieux travailler pendant les études ».

Répondant $\mathrm{n}^{\circ} 22$ :

"Les travaux pratiques sont intéressants, utiles, importants et pertinents. La combinaison de disciplines théoriques et de contenus de stage permet de réaliser une recherche qualitative dans le cadre d'un mémoire de master $»$.

En réponse aux questions sur le problème de l'interaction entre les participants au processus de formation pratique (responsables de stage de l'université, école, étudiants, responsables de l'établissement scolaire), les répondants $(54,48 \%)$ ont noté un niveau de communication suffisamment élevé avec tous les acteurs des pratiques. Parmi les difficultés d'interaction au sein des pratiques, 13,62\% des répondants ont cité le format à distance, en soulignant la nécessité et l'importance de la communication vivante dans la mise en œuvre du processus de formation pratique.

Ainsi, les répondants considèrent la construction du programme d'études selon le principe de la modularité comme une solution efficace à la question de l'intégration de la théorie et de la pratique dans la formation des enseignants avec le potentiel de la continuité du contenu des disciplines théoriques et des pratiques, du dialogue des acteurs de l'activité éducative, ainsi que de la combinaison des outils d'évaluation des disciplines et des pratiques.

\section{Le cas de l'Université nationale eurasienne nommée d'après L.N. Goumilev}

$\mathrm{Au}$ cours des recherches menées, les résultats suivants ont été obtenus.

1. Selon le corps des enseignants de l'université :

$80 \%$ des répondants soulignent l'impact significatif de la pandémie sur le système éducatif et la formation théorique et pratique du personnel enseignant (domaine de l'enseignement des langues). $40 \%$ des répondants ont exprimé un manque d'intérêt pour le processus de maîtrise des compétences des nouvelles technologies numériques nécessaires à la mise en œuvre des formats d'apprentissage à distance, tandis que $70 \%$ reconnaît le fait que l'apprentissage à distance pendant la pandémie est l'un des moyens les plus efficaces pour obtenir les compétences nécessaires à l'exercice d'une profession.

Répondant $\mathrm{n}^{\circ} 7$ :

" Aujourd'hui, un étudiant a la possibilité d'acquérir des connaissances n'importe où et à n'importe quel moment de la journée, lorsque cela lui convient et il peut apprendre ce qu'il aime. L'utilisation d'outils tels que Zoom, Microsoft Teams nous permet de former des enseignants de français ».

Répondant $\mathrm{n}^{\circ} 2$ :

"La période pandémique et le passage au format d'apprentissage à distance sont un défi majeur pour les enseignants et les étudiants. Ici, il est très important de prendre des décisions stratégiques de gestion sur l'application centralisée de certains systèmes d'information à des fins éducatives. Notre université utilise le système Platonus. Ce système permet aux enseignants de télécharger du matériel pédagogique, des rapports, des syllabus, ainsi que d'interagir avec les étudiants via des profils personnels. Il est possible de vérifier très rapidement les connaissances des élèves, de leur fournir un retour d'information, etc. ».

$40 \%$ des répondants pensent qu'ils ont une connaissance insuffisante des technologies numériques pour la mise en œuvre de l'enseignement à distance et combiner de façon satisfaisante les disciplines théoriques et pratiques dans la formation des futurs enseignants. $50 \%$ des répondants ont souligné le manque de temps pour se familiariser avec les nouveaux outils de formation des enseignants (plateformes, applications, etc.).

XLinguae, Volume 14 Issue 4, October 2021, ISSN 1337-8384, eISSN 2453-711X 
Répondant $\mathrm{n}^{\mathrm{o}} 3$ :

"A mon avis, il faut une certaine régularité pour maîtriser des technologies numériques éducatives sélectionnées et planifier leur introduction (et non dans l'urgence!) en tenant compte de la charge de travail des enseignants. A cause du manque de temps, il est très important de planifier intelligemment l'organisation des activités et de réfléchir à l'introduction d'un nouvel outil numérique dans le processus éducatif ».

Répondant $\mathrm{n}^{\circ} 9$ :

"Il serait productif d'organiser un jour par semaine une formation spéciale sur l'une des technologies numériques. En tant qu'enseignants, nous pourrions nous concentrer sur cette tâche précise et notre compétence numérique s'en trouverait grandement améliorée ».

$60 \%$ des répondants ont indiqué que les étudiants sont souvent impliqués dans la recherche de nouveaux moyens de mettre en œuvre le processus éducatif : on leur assigne des travaux pratiques pour la recherche, la maîtrise et la présentation des technologies numériques modernes ainsi que des ressources éducatives utilisées dans l'enseignement à distance dans la préparation des enseignants de français. Ensuite, les résultats de leurs activités sont discutés dans des séminaires et des stages dans le cadre desquels il se produit un échange d'expériences et un enrichissement mutuel avec de nouvelles connaissances concernant les outils méthodologiques numériques.

Répondant $\mathrm{n}^{\mathrm{O}} 10$ :

"Nous appelons cela un assistanat pédagogique "numérique "à l'université : nos étudiants en master, en doctorat et en thèse recherchent le matériel électronique et les programmes nécessaires aux cours des enseignants. Les résultats de la recherche et de l'approbation pratique des outils numériques dans la pratique sont discutés collectivement, leurs avantages et inconvénients sont déterminés, et les possibilités de leur application dans le processus de formation linguistique des futurs enseignants sont établies ».

Ainsi, il est raisonnable de parler de l'impact significatif de la pandémie sur le processus de formation théorique et pratique des enseignants au Kazakhstan (le contexte de l'enseignement linguistique), qui s'est traduit par un passage centralisé à un format d'interaction à distance entre tous les participants au processus éducatif (outils Zoom, Microsoft Teams, Platonus), ce qui a favorisé un apprentissage plus flexible, la capacité de mettre en œuvre l'éducation sur des pistes individuelles (sans lien avec le lieu, en choisissant le moment qui convient à chacun pour étudier les cours théoriques et pour prendre part à la formation pratique). Nous considérons qu'il est important d'impliquer les corps des masters, étudiants en thèse et les doctorants dans le processus de recherche et de test de nouveaux outils numériques (assistanat pédagogique "numérique") et de réfléchir aux résultats de leur utilisation dans la pratique avec le corps enseignant (mise en œuvre du principe de dialogue des acteurs du processus éducatif). Parallèlement aux effets positifs, il a été fait état d'un grand nombre de problèmes touchant à l'organisation de la formation professionnelle des enseignants ayant ce profil linguistique, à savoir : l'absence d'un système complet de numérisation du processus éducatif, le manque de concentration sur une spécialité particulière, le manque aigu de temps des enseignants universitaires pour rechercher et maîtriser les technologies numériques productives, ainsi que le manque de motivation pour la création et l'utilisation des outils numériques et les ressources.

La possibilité d'une intégration réussie de la théorie et de la pratique dans la mise en œuvre de la formation professionnelle d'un futur enseignant de français à l'université est évaluée positivement par $60 \%$ des enseignants interrogés. $40 \%$ des répondants ont souligné la priorité de l'utilisation de simulateurs, d'enregistrements audio, de fragments vidéo lors de la formation des compétences phonétiques, lexicales et grammaticales, mettant l'accent sur l'importance de la perception polymodale des phénomènes linguistiques (principe de polymodalité). Lors des entretiens avec les 
enseignants interrogés, des applications web telles que « 6000 Words - Learn French Language for Free », «Learn French by Bravolol Limited », «Learn French with Babbel », «Le Conjugueur » utilisées activement dans les universités du Kazakhstan pour la formation des enseignants ont été discutées. La plus populaire d'entre elles est l'application «6000 Words - Learn French Language for Free », $100 \%$ des répondants l'utilisent dans leurs activités d'enseignement. La deuxième application la plus populaire est «Learn French with Babbel » $60 \%$ des répondants $)$, la troisième est « Learn French by Bravolol Limited » (40\% des répondants), la dernière application en termes de popularité est « Le Conjugueur» (20\% des répondants).

Répondant $\mathrm{n}^{\circ} 8$ :

"J'utilise l'application de conjugaison des verbes français lors de la mise en ouvre des cours pratiques. La base de données compte plus de 9000 verbes différents, ce qui permet à l'étudiant d'obtenir immédiatement la conjugaison complète d'un verbe français en utilisant le moteur de recherche très performant; l'application a accès à près de 10000 tableaux de conjugaison de verbes...".

Répondant $\mathrm{n}^{\mathrm{0}} 3$ :

«Cette application est adaptée à l'utilisateur spécifique : pendant les tâches et les tests, l'application Web vous proposera exactement les mots avec lesquels vous avez déjà eu des problèmes. Par exemple, si vous faites une erreur dans la définition du sens d'un mot, ce même mot vous sera proposé à nouveau les fois suivantes jusqu'à ce que vous vous en souveniez. De plus, le processus d'apprentissage est accompagné d'illustrations colorées, de transcriptions phonétiques et d'enregistrements de la prononciation correcte de locuteurs natifs. Le graphisme et le son se combinent pour renforcer la perception du mot de la langue étrangère, le processus de mémorisation est plus efficace ».

Répondant $\mathrm{n}^{\circ} 8$ :

«Ici, il y a une fonction intéressante, l'enregistrement de la voix, avec laquelle il semble possible de vérifier si votre prononciation est correcte ».

L'évaluation positive des offres Web par les enseignants dans la mise en œuvre de la formation des futurs enseignants de FLE s'accompagne de l'indication de la nécessité ( $30 \%$ des répondants) d'analyser le potentiel didactique d'un outil numérique ou d'une ressource avant de l'utiliser dans la pratique.

Répondant $\mathrm{n}^{\circ} 1$ :

«Pendant la formation pratique, nous commençons à maîtriser la technologie numérique par une introduction théorique à celle-ci. Les étudiants se familiarisent avec le matériel méthodologique, étudient le potentiel de la technologie numérique, analysent ses avantages et ses inconvénients, essaient de l'appliquer dans la pratique. Une fois qu'ils maîtrisent suffisamment cette technologie, ils la présentent à des étudiants de première année et à des enseignants travaillant avec eux dans des disciplines théoriques. Une telle interaction est utile à tous, car elle permet de se familiariser avec la technologie numérique testée dans un laps de temps assez court ». Concernant la question de la combinaison des disciplines théoriques et de la pratique dans l'enseignement à distance, la majorité $(80 \%)$ des répondants en souligne la possibilité. Cependant, $60 \%$ des répondants plaident en faveur de l'apprentissage mixte comme format prioritaire pour la mise en œuvre de la formation théorique et pratique des futurs enseignants de français. En particulier, les enseignants voient une difficulté concrète dans le développement du discours oral en ligne en langue étrangère : assez souvent, dans l'enseignement supérieur, la méthode de communication directe entre deux ou plusieurs interlocuteurs est utilisée à cette fin.

Répondant $\mathrm{n}^{\mathrm{o}} 10$ :

"Le développement du langage oral implique une communication vivante. Bien sûr, il peut aussi être organisé en ligne... Cependant, dans ce cas, les étudiants n'ont pas la

XLinguae, Volume 14 Issue 4, October 2021, ISSN 1337-8384, eISSN 2453-711X 
possibilité de saisir les expressions faciales et les gestes, de percevoir la cinétique du corps dans le processus de communication, donc la réaction à la réplique n'est pas tout à fait correcte, il y a un malentendu, la logique de l'acte de communication est rompue ».

En conséquence, le problème de l'intégration des disciplines théoriques et de la pratique lors de la formation d'un futur professeur de FLE dans les établissements d'enseignement supérieur kazakhs dans des conditions pandémiques est associé au respect du principe de polymodalité (multimédia). Le principe du dialogue des acteurs $\mathrm{du}$ processus éducatif, mis en œuvre sous la forme d'un assistanat pédagogique "numérique", joue également un rôle particulier dans ce domaine. Dans la pratique, on constate une augmentation de l'indépendance du corps étudiant. La position dominante parmi les enseignants est la priorité du format d'apprentissage mixte, l'impossibilité de remplacer la communication vivante comme principal moyen de développer le discours oral en langue étrangère. Les problèmes les plus aigus de la formation professionnelle théorique et pratique d'un professeur de français dans les universités du Kazakhstan sont les suivants: l'absence d'un système complet de numérisation du processus éducatif, le manque de temps pour que les enseignants apprennent à maîtriser les technologies numériques, leur manque de compétence dans le domaine des nouveaux outils d'apprentissage à distance et, par conséquent, leur faible motivation pour développer et tester dans la pratique leurs propres ressources pédagogiques numériques.

2. Selon le corps des étudiants de l'université (étudiants en master) :

$77,18 \%$ des étudiants interrogés notent l'impact significatif de la pandémie sur le domaine de l'éducation. $63,56 \%$ des répondants soulignent les avantages de l'enseignement à distance. Un certain nombre d'étudiants $(22,7 \%)$ relèvent l'évaluation positive que font les parents du format numérique de l'enseignement.

Répondant $\mathrm{n}^{\circ} 21$ :

"Pour moi, qui suis mère de jeunes enfants, le format d'apprentissage à distance me semble extrêmement utile. Grâce à ce système, j'ai pu poursuivre mes études et ne pas les interrompre pendant les vacances. "

Répondant $\mathrm{n}^{\circ} 16$ :

"Pendant la pandémie, nous étions déconnectés de notre université et n'avions pas la possibilité de communiquer en face à face, "en direct», avec nos professeurs. D'autre part, beaucoup d'entre nous pouvaient étudier au domicile de leurs parents et les aider dans les tâches ménagères pendant leur temps libre ».

Répondant $\mathrm{n}^{\mathrm{o}} 3$ :

" Grâce à la possibilité d'étudier dans un format d'enseignement à distance, j'ai pu entamer une deuxième formation supérieure dans le cadre d'un programme de mobilité universitaire. En travaillant depuis un seul endroit, j'ai pu poursuivre deux objectifs éducatifs, ce qui m'a permis d'économiser mon temps et mes effort ».

Lorsqu' à été abordée la question de l'intégration réussie de la théorie et de la pratique dans un environnement d'enseignement à distance au cours des entretiens avec les étudiants, $59,02 \%$ des interrogés ont expliqué qu'ils considéraient cette intégration comme possible. $54,48 \%$ des répondants ont souligné la priorité du format d'apprentissage mixte dans la mise en œuvre des programmes de formation professionnelle théorique et pratique pour les futurs enseignants de français.

Répondant $\mathrm{n}^{\circ} 8$ :

"Je pense qu'un certain nombre de questions théoriques ainsi que l'analyse des programmes et des outils de formation peuvent être maîtrisés en ligne. En même temps, le processus de planification des activités professionnelles exige une discussion constante du processus dans le groupe, en format hors ligne ».

Répondant $\mathrm{n}^{\circ} 19$ :

«Il y a un certain nombre de problèmes que je ne peux pas résoudre à distance: Comment choisir la meilleure technique pédagogique parmi différentes options? 
Comment puis-je vérifier le temps d'apprentissage dont je disposerai pour une certaine étape du cours? Je pense que je ne peux pas trouver de réponse à ces questions lorsque je travaille à distance... ».

Répondant $\mathrm{n}^{\mathrm{o}} 7$ :

«Dans la pratique, il est très important d'obtenir un retour d'information : de l'enseignant, des autres étudiants et du responsable de stage de l'établissement scolaire. Une discussion de vive voix des résultats de la pratique dans l'équipe m'est beaucoup plus utile».

L'interaction entre le corps étudiant et les responsables de la formation pratique a également été abordée au cours de cette étude. $40,86 \%$ des répondants sont favorables au fait que le format à distance permet une interaction efficace avec le corps enseignant. $45,4 \%$ des étudiants interrogés a admis que l'expérience de travail autonome avec les nouveaux outils numériques initiée par les professeurs de l'université leur a permis de développer des compétences en termes de maîtrise des TIC. Cependant, la norme de présenter publiquement un outil numérique maîtrisé et testé a été un véritable défi pour $36,32 \%$ des répondants et leur a demandé plus d'efforts de préparation. $77,18 \%$ des interrogés notent le fait qu'ils sont devenus beaucoup plus compétents dans l'utilisation des outils numériques modernes. Dans le même temps, $68,1 \%$ des répondants ont déclaré n'être pas suffisamment intéressés par un apprentissage autonome du français en utilisant les technologies numériques dans un format d'apprentissage à distance.

Au cours de cette étude, les étudiants ont également discuté de leurs connaissances et de leurs compétences avec des applications Web multimédia telles que « 6000 Words - Learn French Language for Free », «Learn French by Bravolol Limited », « Learn French with Babbel », «Le Conjugueur». 100\% des répondants ont exprimé leur connaissance et leur compétence sur l'application « 6000 Words - Learn French Language for Free » et «Learn French with Babbel» (100\% des répondants), les applications classées en deuxième lieu sont «Learn French by Bravolol Limited » (40,86\% des répondants), et « Le Conjugueur » (40,86\% des répondants).

De fait, lorsqu'ils définissent le format à distance comme possible et, dans une certaine mesure plus adapté, pour la mise en œuvre de la formation professionnelle du futur professeur de FLE, les élèves de master de l'établissement d'enseignement supérieur kazakh mettent un accent particulier sur l'importance de combiner les formats d'enseignement en ligne et hors ligne (apprentissage mixte) pour une meilleure intégration de la théorie et de la pratique. L'organisation du travail indépendant en relation avec la recherche et le test des technologies numériques modernes en interaction avec les enseignants, ainsi que l'introduction dans le processus d'apprentissage d'applications Web multimédia visant la perception polymodale permettent de conclure à la construction du processus éducatif en accord avec les principes du dialogue des acteurs de l'éducation, la polymodalité.

\section{Le cas de l'Université Comenius de Bratislava}

En République slovaque, il a été démontré que la partie la plus difficile de la formation des enseignants dans les facultés pédagogiques est l'offre de pratiques pédagogiques. Il s'agit notamment de former des professeurs de langues étrangères. La pratique de l'enseignement à distance du point de vue de l'enseignant est difficile à préparer. Créer des activités, planifier du temps et surtout le stress lié au fait que la technologie fonctionnera comme il se doit et que la connexion Internet sera suffisante. Du point de vue de l'étudiant, cependant, ce n'est pas plus facile et moins stressant. C'est la vérification de ces situations d'enseignement et de formation dans l'environnement en ligne qui aidera les enseignants et les étudiants à avoir à l'avenir cette vision factuelle de la situation.

XLinguae, Volume 14 Issue 4, October 2021, ISSN 1337-8384, eISSN 2453-711X 
La pratique pédagogique au cours de l'année universitaire 2020/2021 dans les facultés préparant les enseignants en République slovaque consistait généralement à enseigner des cours en ligne à des enseignants de plusieurs écoles secondaires et à des webinaires de plusieurs invités. Il s'agissait, par exemple, du personnel pédagogique, des psychologues scolaires, du personnel du Centre de conseil et de prévention pédagogiques et psychologiques, y compris des experts étrangers. Tous présentés plustôt sous la forme de conférences supplémentaires à l'étude. Nous résumons les résultats d'une enquête menée auprès de 11 étudiants se préparant à la profession d'enseignant à la Faculté d'éducation de l'Université Comenius de Bratislava (Hradská, Kohútová, Plavčan, Ševčíková, 2021).

Points positifs de la pratique pédagogique à distance terminée :

- Familiarisation avec plusieurs applications en ligne qui peuvent aider les étudiants et les enseignants à l'avenir et diversifier les cours dans l'espace en ligne, ainsi que dans l'enseignement à temps plein.

- Utilisez ces applications en ligne pour convertir les commentaires verbaux en commentaires écrits en quelques secondes. De cette façon, l'ensemble du groupe d'étudiants instruits a la possibilité de s'exprimer. Il n'y a pas une telle possibilité dans l'enseignement à temps plein en termes de temps.

- Notre stress dans l'espace en ligne a également diminué. Nous ne nous sommes soudainement pas sentis nerveux au sujet des élèves ou des étudiants qui sont les principaux acteurs du processus d'enseignement. Nous n'avions pas l'"adrénaline" positive de nous tenir devant la salle de classe et de prendre le contrôle de son espace.

- Cette façon d'enseigner correspond également les compétences du 21 ème siècle.

- L'approche des enseignants en exercice, qui ont très bien accompagné cette pratique pédagogique en ligne exigeante.

- Enfin et surtout, la possibilité d'essayer d'apprendre, même si ce n'est que dans un environnement en ligne.

Négatifs de la pratique pédagogique à distance terminée

- Il n'y a pas assez d'interaction de la part des élèves, que ce soit par ex. Je comprends le sujet.

- L'enseignant ne peut pas vérifier si les élèves sont assis derrière les écrans d'ordinateur et écoutent attentivement, ou s'ils ont un son en sourdine et font « leurs » activités.

- Les excuses pour une heure ne sont pas pour des raisons telles que : je me suis endormi, je ne me suis pas préparé, mais elles le sont, par exemple, pour des raisons telles que : je n'ai pas de technique, mon micro ou ma caméra est cassé.

- Il n'y a aucune possibilité de communication non verbale. Le contact direct dans les pratiques pédagogiques vous permet de développer des compétences de communication suffisantes ainsi qu'un sens de la présentation en classe.

- Incapacité à gérer des situations éducatives avec divers problèmes en classe.

- La capacité limitée d'essayer d'être dans la salle de classe pour les élèves et d'un point de vue humain en tant que modèle, pas seulement une " encyclopédie » sur un écran d'ordinateur.

- Être placé devant toute la classe, où l'on peut voir ce qui s'y passe, comment la classe « vit » et comment l'enseignant « s'y intègre ».

- Le stress de la technologie, qui est censée être un moyen de nous aider. Ces fonctions ont été échangées, et la technique est ainsi devenue un facteur de stress et les élèves ceux qui ont aidé à la maîtriser.

- Capacité limitée à faire preuve de «maîtrise de la parole » devant le public cible, c'est-à-dire devant les élèves de la classe, pour sortir d'une certaine zone de confort et ne pas se cacher derrière des écrans d'ordinateur.

- La préparation d'une leçon en ligne prend avant tout beaucoup de temps pour rendre la leçon interactive et visuelle. 
- Elaboration de tâches non adaptées à l'enseignement en ligne, par exemple remplir un portfolio contenant des tâches telles que «Observer et identifier (décrire brièvement) des situations dans lesquelles les étudiants étaient inattentifs et flous, resp. n'a pas montré d'intérêt pour l'enseignement. » Les élèves ne sont pas visibles dans l'enseignement en ligne. Ils doivent être activement impliqués dans cet enseignement en ligne par le biais de questions.

\section{Conclusion}

Cette étude comparative de cas d'universités de trois pays, réalisée sur la base d'approches systémique et actionnelle, montre l'efficacité de l'organisation du processus de formation professionnelle d'un futur enseignant de FLE en situation de pandémie par l'intégration de la théorie et de la pratique au niveau des contenus et des technologies d'apprentissage. Ceci est favorisé par : 1. l'application de programmes d'études flexibles (principe de modularité) ; 2. l'utilisation des modes de perception visuels, auditifs et moteurs au moyen de technologies numériques dans le processus éducatif (principe de multimodalité (multimédia) de l'apprentissage) ; 3. la continuité du contenu des disciplines et des pratiques (principe de caractère systémique) ; 4 . l'interaction et la communication efficaces de tous les acteurs du processus éducatif (principe de dialogue). Un rôle particulier est joué par le système d'évaluation intégrée du niveau des compétences à condition de le combiner avec les fonds d'outils d'évaluation des disciplines et des pratiques (principe d'enchaînement). L'étude a révélé les principes dominants de modularité, de régularité, de dialogue et d'enchaînement dans le cas institutionnel de l'Université Fédérale de Kazan. Pour l'étude des cas de l'Université nationale eurasienne nommée d'après L.N. Goumilev et de l'Université Comenius de Bratislava les principes de polymodalité (multimédia) et de dialogue se sont avérés déterminants. Les résultats de l'étude peuvent être utilisés dans le développement et la modification des programmes d'études, des programmes de travail, dans la recherche comparative sur la formation des enseignants afin d'identifier les possibilités d'enrichissement mutuel de la formation théorique et pratique des futurs enseignants de français dans différents pays (Cabassut , Ferrando, 2017).

\section{Remerciements}

Ce travail a été réalisé à la charge du budget du programme stratégique de leadership académique de l'Université fédérale de Kazan (région de la Volga). Les auteurs remercient les professeurs de français et les étudiants du département de philologie étrangère de l'Université nationale eurasienne nommée d'après L.N. Goumilev, et ceux de l'Université fédérale de Kazan (région de la Volga), les étudiants de la Faculté d'éducation de l'Université Comenius de Bratislava pour leur aide dans la collecte des informations factuelles, ainsi que l'Académie nationale de l'éducation nommée d'après Y. Altynsarin, qui a été la base de recherche pour le dépouillement des matériaux factuels obtenus.

\section{Bibliographic references}

ALSHTAIWI, M. 2020. Extraction des termes sur la COVID-19 et leurs emplois sémantico-syntaxiques à partir d'un corpus spécialisé. In: Synergies Algérie. ISSN 1961-9472

ASLANYAN, A.S. 2021. Features of distance learning in English during the COVID19 pandemic. In Pedagogy, Psychology, and Society: a New Reality, pp. 105-108. ISBN 978-5-907411-03-6.

ASSUNÇÃO FLORES, M. - GAGO, M. 2020. Teacher education in times of Covid19 pandemic in Portugal: national, institutional and pedagogical responses. In: 
Journal of Education for Teaching, vol. 46, n. 4, pp. 507-516. ISSN 1360-0540, 0260-7476.

AUBRY, A. 2020. Les doubles certifications européennes : le cas du Bachibac en Andalousie. In: Synergies Europe, Vol. 15, pp. 181-190. ISSN 1951-6088 ISSN en ligne 2260-653X

AUGER, N. - LOUIS, V. - DERVIS, F. 2009. CECR and intercultural dimension of FLE teaching / learning: what tasks possible? In: French in the World, vol. 45, pp. 103-110.

BABENKO, O. - ABROSIMOVA, N. - PROSYUKOVA, K. - FOMINYKH, A. 2017. Teaching first-year students collaboration skills and shaping academic readiness in multi-cultural society//INTED2017: 11th International technology, education and development conference. Valencia, Spain, 6-8 March, 2017. In: Conference proceedings. pp. 4979-4985. ISSN: 2340-1079.

BAGHAHA, J. - VOLOSHINA, T.G. - GLEBOVA, YA. A. - BOCHAROVA, E.A. - RADOVICH, M.A. 2020. Globalization influence on linguistic and cultural state due to the language contacts' interaction. In: Laplage em Revista (Sorocaba). vol. 6, pp.190-197.

BAKLASHOVA, T. - SKOBELTSYNA, E.G. - GALISHNIKOVA, E.M. 2020. Developing Teacher Competences: Key Issues and Values. Modification of Practical Training Programs in Pedagogical Master Courses: In Pursuit of Professional Development. New York: Nova Publishers, 353 p. ISBN: 978-1-53618-227-9.

BAKLASHOVA, T.A. - SAKHIEVA, R.G. - TELEGINA, N.V. - SKOBELTSYNA, E.G. - BABIEVA, N.S. 2020. Design of Praxis Programs for Students of Pedagogical Master's Degree Studies in The Context of Federal State Educational Standards Implementation. In: Talent Development \& Excellence, vol. 12, pp. 153-162. ISSN: 1869-0459.

BAYNAT MONREAL, E. - LÓPEZ, M. 2020. La machinima Entrevista de trabajo / Entretien d'embauche : une ressource numérique pour l'apprentissage de la langue française en contexte universitaire. In: Synergies Europe, Vol.15, pp. 163-179. ISSN 1951-6088

BENITO PEREZ, S. 2020. L'enseignement à distance. Proposition didactique pour l'enseignement du FLE. Universidad de Valladolid. Facultad de Filosofía y Letras. Available online: https://uvadoc.uva.es/bitstream/handle/10324/45903/ tfm_f_2020_87.pdf?sequence=1\&isAllowed=y.

BOVTENKO, M.A. 2005. Formation of the information and communication component of the professional competence of a foreign language teacher. In: Bulletin of the Moscow University. Series 19. Linguistics and Intercultural communication, vol. 4, pp. 54-67. ISSN 0130-0105.

BULATBAEVA, K. - SIVAKOVA, I. - GALIAKHMETOVA, A. 2017. Cognitivecommunicative technology as a mechanism for the formation of subject-oriented foreign language competence of students. In: Foreign languages in the modern world pp.100-106. Available online: https://www.elibrary.ru/download/elibrary_30217704 _27881295.pdf.eLIBRARY ID: 30217296.

CABASSUT, R. - FERRANDO, I. 2017. Difficulties in Teaching Modelling: A French-Spanish Exploration. In: Stillman G., Blum W., Kaiser G. (eds). Mathematical Modelling and Applications. International Perspectives on the Teaching and Learning of Mathematical Modelling. Cham: Springer. ISBN 978-3-319-62967-4.

CUTRI, R.M. - MENA, J. - WHITING, E. 2020. Faculty readiness for online crisis teaching: transitioning to online teaching during the COVID-19 pandemic. In: European Journal of Teacher Education, vol. 43, n. 4, pp. 523-541. ISSN 0261-9768. DUMONT, E. 2019 Apprivoiser la distance en cours particuliers de français langue étrangère par videoconference. In: Synergies France, Vol. 13, pp. 169-182. ISSN 1766-3059 
EFIMKINA, E.A. 2010. Methods of using distance learning technologies in teaching French to linguist students: Doctoral Dissertation. Nizhny Novgorod: Nizhny Novgorod State University named after N.A. Dobrolyubov.

ERLAM, R. - ELLIS, R. 2018. Task-Based Language Teaching for Beginner-Level Learners of L2 French: An Exploratory Study. In: The Canadian Modern Language Review, vol. 74, n. 1, pp. 1-26. ISSN 0008-4506.

ERSIN, P. - ATAY, D. 2021. Exploring online mentoring with preservice teachers in a pandemic and the need to deliver quality education. In: International Journal of Mentoring and Coaching in Education, vol. 10, n. 2, pp. 203-215. ISSN 20466854, 20466862.

FAHRUTDINOVA, G.Z. - BULATBAYEVA, K.N. - KONDRATOVA, O.I. FEDOROVA, S.N. - PETUKHOVA, O.A. 2018. Updating of language training content in Kazakhstan: implementation of new linguodidactic approaches. In: XLinguae, vol. 11, n. 2, pp. 239-255. ISSN 1337-8384.

GAFUROV, I. R. - VALEEVA, R. A. - KALIMULLIN, A. M. - SAKHIEVA, R. G. 2018. Testing Practice-Oriented Master's Programme in "Education and Pedagogical Sciences" (Teacher of Basic General Education). In: Psychological Science and Education, vol. 23, n. 1, pp. 25-37. ISSN: 1814-2052 / 2311-7273 (online).

GAO, L.X. - ZHANG, L.J. 2020. Teacher learning in difficult times: Examining foreign language teachers' cognitions about online teaching to tide over COVID-19. In: Frontiers in Psychology, vol. 11, 2396. ISSN 1664-1078.

GARTSOV, A.D. 2009. Electronic linguodidactics in the system of innovative language education: doctoral dissertation. Moscow: Peoples' Friendship University of Russia.

GRIFTSOVA, I.N. - LINKOV, V.V. 2018. On the relationship between theory and practice in education: socio-cultural factors and their impact on the educational process. In: Personal development, vol. 2, pp. 13-25. ISSN 2073-9788.

HRADSKA, L. - KOHUTOVA, K. - PLAVCAN, P. - SEVCIKOVA, A. 2021. Dopad pandemickej situácie na pedagogicku prax na fakultach pripravujucich ucitelov [The impact of the pandemic situation on pedagogical practice at teacher training faculties]. In: Revue spolocenskych a humanitnych vied. ISSN 1339-259X

KANSEEL, R. 2020.La philologie française et son impact sur la didactique du français langue-culture étrangère du point de vue des professeurs universitaires. In: Synergies Europe, vol. 15, pp. 71-84. ISSN 1951-6088 ISSN en ligne 2260-653X

KAPITSA, A. 2013. Computer technologies as a means of teaching a foreign language in institutions of higher education. In: Bulletin of the Kazakh-American Free University. General problems of philology, vol. 2, pp. 158-161. ISSN 18159478.

KAVILOVA, T. 2020. Teaching dialogic speech in french classes. In: JSPI Scientific Publications Archive, vol. 23, n. 1. Available online: https://science.iedu.uz/index.php/archive_jspi/article/view/1549. ISBN: 978-19997898-5-5.

KASPARY, C.V. - DE SOUSA, C.P. 2020. La conception d'un cours de formation d'enseignants dans le contexte de FLE. In: Revista Letras Raras, vol. 9, n. 5, pp. 4462. ISSN: 2317-2347

KHASANOVA, G.A. 2021. Methodology of Teaching French. Archive of Conferences. $1 . \quad$ Retrieved from https://conferencepublication.com/index.php/aoc/article/view/548

KOSTIKOVA, L.P. 2008. Modern education from the standpoint of the dialog approach. In: Education and Science, vol. 4, pp. 3-13. ISSN 1994-5639, 2310-5828

KOSYAKOVA, L.N. - POPOVA, A.L. 2020. Distance education in an agricultural university: pros and cons. In: Large Conference Hall: additional education-vectors of development, pp. 37-44. ISSN 2618-7310.

XLinguae, Volume 14 Issue 4, October 2021, ISSN 1337-8384, eISSN 2453-711X 
LA VELLE, L. - NEWMAN, S. - MONTGOMERY, C. - HYATT, D. 2020. Initial teacher education in England and the Covid-19 pandemic: challenges and opportunities. In: Journal of Education for Teaching, vol. 46, n. 4, pp. 596-608. ISSN 0260-7476, 1360-0540.

MARINONI, G. - LAND, H. - JENSEN, T. 2020. The Impact of COVID-19 on Higher Education around the World. Paris: International Association of Universities, UNESCO House. ISBN 978-92-9002-212-1

MARTIN, J.A.S.C. 2020. Les rôles des participants à un projet télécollaboratif en français entre deux universités européennes. In: Synergies Europe, Vol.15, pp. 145161. ISSN 1951-6088 ISSN en ligne 2260-653X

MENTER, I. - VALEEVA, R.A. - KALIMULLIN, A.M. 2017. A tale of two countries - forty years on: politics and teacher education in Russia and England. In: European Journal of Teacher Education, vol. 40, n. 5, pp. 616-629. ISSN 1469-5928, 0261-9768.

MIKHAILOVA, T. - DUISEKOVA, K. - ORAZAKYNKYZY, F. BEYSEMBAEVA, G. - ISSABEKOVA, S. 2021. The evaluation of intercultural professional technology-based communicative competence formation for students. In: World Journal on Educational Technology: Current issues, vol. 13, n. 2, pp. 272-287. ISSN 1309-0348, 1309-1506.

MISHRA, L. - GUPTA, T. - SHREE, A. 2020. Online teaching-learning in higher education during lockdown period of COVID-19 pandemic. International Journal of Educational Research Open, 1, 100012. ISSN: 2666-3740.

MORVAN, Y. - FRAJERMAN, A. 2021. La santé mentale des étudiants: mieux prendre la mesure et considérer les enjeux. In: L'encéphale. ISSN 0013-7006, https://doi.org/10.1016/j.encep.2020.10.009. Available online: https://www.sciencedirect.com/science/article/pii/S001370062100035X).

MURPHY, L. - EDULJEE, N.B. - CROTEAU, K. 2020. College Student Transition to Synchronous Virtual Classes during the COVID-19 Pandemic in Northeastern United States. Pedagogical Research, 5(4), em0078. https://doi.org/10.29333/pr/8485. e-ISSN: 2468-4929.

NOZDREVATYKH, B.F. - NOZDREVATYKH, D.O. 2020. Modularity in education. In: Proceedings of the international scientific and methodological conference, 31 January 2020 Russia, Tomsk, pp. 93-94. ISBN: 978-5-86889-859-4.

OECD. 2020. Social Economy and the COVID-19 Crisis: Current and Future Roles. Paris: Organization for Economic Cooperation and Development. oecd.org/coronavirus/

NOMOKONOVA, O.YU. - TSVETKOV, V.YA. 2015. A systematic approach in scientific research. In: Slavic Forum, vol. 2, pp. 224-232.

PODYMOVA, L.S. - GOLOVYATENKO, T.A. - KHMELKOVA, M.A. 2021. Innovative technologies in the management system of digital education in the conditions of higher education. In: Higher Education Today, vol. 2, pp. 12-16. ISSN: 1726-667X.

PRUVOST, V.I. - NADIM, L.N. 2020. Les rives identitaires de Leïla Houari: le récit d'une femme tiraillée entre tradition et modernité. In: Synergies Europe, 2020, Issue 15, pp.99-112.

ROY, M. 2021. Les relations dans la communauté scolaire à l'heure de la Covid 19: évolution de la communication entre élèves, parents et professeurs. In: Synergies Pays germanophones, vol. 14, pp. 119-137. ISSN 1866-5268

SAADATNEJAD, Z. - FARSIAN, M.R. 2020. Improving Intercultural Competence through Literary Texts and the Strategies of Teaching it in French Language. In: Tarbiat Modares University Press, vol. 10, n. 6, pp. 345-372. ISSN 0210-6124.

SANCHEZ-GARCIA, M.J. - GUANLIN, H. - PINTO ESCRIBANO, J. 2013. Teacher Development and ICT: The Effectiveness of a Training Program for In- 
service School Teachers. In: Procedia - Social and Behavioral Sciences, vol. 92, pp. 529-534. ISSN 1877-0428.

SEROSTANOVA, N.N. - CHOPOROVA, E.I. 2020. Modern technologies of teaching foreign languages in the era of digitalization of education. In: Modern Problems of science and Education, vol. 6, pp. 10-10. ISSN 2070-7428.

SIROTOVA, M. - MICHVOCIKOVA, V. 2021. Virtual Reality - Part of Supervised Teaching Practice for University Students - Future Teachers? In: European Journal of Contemporary Education, vol. 10, n.1, pp. 127-136.

SIROTOVA, M. - MICHVOCIKOVA, V. - HOSTOVECKY, M. 2021. Serious Games in University Education of Future Teachers. Berlin: Peter Lang. 220 p. ISBN 978-363184832-6, 978-363184833-3

SHAHRILL, M. - PETRA, M. I. - NAING, L. - YACOB, J. - SANTOS, J.H. AZIZ, B.A. 2021. New norms and opportunities from the COVID-19 pandemic crisis in a higher education setting: Perspectives from University Brunei Darussalam. In: International Journal of Educational Management, vol. 35, n. 3, pp. 700-712. ISSN: 0951-354X.

SHEPTUKHOVSKY, M.V. 2012. Activity-based approach in pedagogical research. In: Scientific Search, vol. 2, n. 6, pp. 26-27. ISSN 2224-6436.

SMUK, M. 2020. Études françaises à l'Université de Varsovie (1919-2019). Du contexte local aux défis universels. In: Synergies Europe, vol. 15, pp.19-32. ISSN 1951-6088 ISSN en ligne 2260-653X

SOLOVYEVA, E. - SABIROVA, D. - MOROZOVA, T. 2015. Forming students' linguistic and cultural competence in academic translation and interpretation studies. In: Procedia-Social and Behavioral Sciences, vol. 199, pp. 415-422. doi:10.1016/j.sbspro.2015.07.527.

SOMKIN, A.A. 2008. System approach and actual problems of modern education (socio-philosophical analysis). In: Integration of education, vol. 2, pp. 107-111. ISSN: 1991-9468.

TRUBAVINA, I. - DOTSENKO, S. - NABOKA, O. - CHAIKOVSKYI, M. MESHKO, H. 2021. Developing digital competence of teachers of Humanitarian disciplines in the conditions of COVID-19 quarantine measures. In: Journal of Physics: Conference Series, vol. 1840, n. 1, 012052. IOP Publishing. ISSN 17426588, 1742-6596.

URIBE GUINARD, S. 2018. La multi-modalité sensorielle pour l'encouragement de la production orale en FLE: Au-delà du symbole. Bogotá: Universidad Pedagógica Naciona. Available online: http://repository.pedagogica.edu.co/bitstream/ handle/20.500.12209/1-1485/TE-22625.pdf?sequence=1\&isAllowed=y

USAK, M. - MASALIMOVA, A. R. 2019. Mentoring on science teacher education in Russia and international perspectives. In: Journal of Baltic Science Education, vol. 18, n.3, pp. 320-322. ISSN 1648-3898, ISSN 2538-7138 (Online).

VALEEVA, R. - GAFUROV, I. 2017. Initial teacher education in Russia: connecting practice, theory and research. In: European Journal of Teacher Education, vol. 40, n. 3, pp. 342-360. ISSN: 0261-9768 (Print), 1469-5928 (Online).

VALEEVA, R.A. - KALIMULLIN, A.M. 2019. Learning to Teach in Russia: A Review of Policy and Empirical Research. In: Knowledge, Policy and Practice in Teacher Education: A Cross-National Study, pp. 193-212. ISBN 978-1-3500-6868-1 (hardback), 978-1-3500-6871-1 (online).

ZERVA, A. 2020. Les études françaises en Grèce. In: Synergies Europe, vol. 15, pp. 47-57. ISSN 1951-6088 ISSN en ligne 2260-653X

Words: 12595

Characters: 85821 (47,71 standard pages) 
Associate Prof. Tatiana Alexandrovna Baklashova, $\mathrm{PhD}$

Institute of Psychology and Education

Kazan Federal University

18, Kremlevskaya Str.

420008 Kazan

Russia

ptatyana2011@mail.ru

Prof. Kulzhanat Nurymzhanovna Bulatbayeva,

Chief Researcher, National Academy of Education named after Y. Altynsarin,

L.N. Gumilyov Eurasian National University,

Nur-Sultan, Kazakhstan

Mangilik El Avenue 8,

Business Center "Altyn Orda", floor 13 - 16

010000 Astana

Kazakhstan

kulzhanat.bulatbayeva@mail.ru

Assoc. Prof. Juan José Mena Marcos, Dr.

Head of Education Department

Faculty of Education

University of Salamanca

$\mathrm{P}^{\mathrm{o}}$ de Canalejas, 169, Office: 38.

37008, Salamanca

Spain

juanjo_mena@usal.es

Treasurer of ISATT (International Study Association on Teachers and Teaching)

Research Officer

Institute of Psychology and Education

Kazan Federal University

18, Kremlevskaya Str.

420008 Kazan

Russia

Prof. Ing. Peter Plavčan, PhD.

Faculty of Social Studies

Danubius Academy

Richterova 1171

92521 Sládkovičovo

Slovak Republic

peter.plavcan@vsdanubius.sk 